\title{
LA PERMANENCIA ESCOLAR EN LAS REDES EDUCACIONALES DESDE COMUNIDADES PERIFÉRICAS
}

\author{
Student Retention within Education Networks from Marginal Communities
}

A permanência escolar nas redes educacionais desde comunidades periféricas

RECIBIDO:27 DE ENERO DE 2014

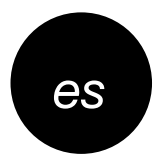

\section{RESUMEN}

Se aborda la influencia del sistema simbólico comunitario en relación con la incidencia de los procesos culturales comunitarios en los procesos educacionales, fundamentalmente en las comunidades de Raposo, Primero de Mayo y Sur Isleta, ya que la reproducción cultural que tiene lugar en ellas permite la persistencia de normas que pueden ser mal interpretadas por otros adolescentes o los mismos profesores del área. Se realiza un análisis de los principales códigos culturales que se encierran dentro del lenguaje y los vocablos que utilizan estos jóvenes como son las normas de convivencia grupales, la percepción estética de la vida cotidiana, señas de identidad comunes, representaciones sociales y prejuicios, estilos de participación social, además de los gestos, las representaciones sociales y esquematizadas. También se han identificado los factores socioculturales que promueven o estimulan el abandono escolar dentro del análisis general y se han agrupado en internos (personales) y externos (grupales e institucionales).

PALABRAS CLAVE: sistema simbólico comunitario, capital simbólico, factores socioculturales, permanencia escolar.

\section{EVALUADO:28 DE ABRIL DE 2014}

Jónathan Jesús Leyva Noa (Cuba)

Máster en Historia y Cultura

Universidad de Guantánamo

jonathan@fcs.cug.co.cu

\section{ACEPTADO:6 DE JUNIO DE 2014}

\section{en}

\section{ABSTRACT}

This article deals with the influence the community symbolic system has regarding the incidence of community cultural processes within educational ones -especially in Raposo, Primero de Mayo, and Sur Isleta- since cultural reproduction in those areas enables the persistence of regulations that can be misinterpreted by teenagers and even teachers. We analyzed the main cultural codes included in the language as well as the vocabulary used by youngsters such as: group communal-living rules, aesthetic perception of daily life, common identity traces, social representations and prejudices, social participation styles, gestures, and social and outlined representations. We also identified (within the general analysis) the socio-cultural factors that promote or encourage attrition, grouping them by internal (personal) and external (group and institutional) aspects.

KEYWORDS: community symbolic system, symbolic capital, socio-cultural factors, student retention. por

\section{RESUMO}

Aborda-se a influência do sistema simbólico comunitário em relação com a incidência dos processos culturais comunitários nos processos educacionais, fundamentalmente nas comunidades de Raposo, Primero de Mayo e Sur Isleta, já que a reprodução cultural que tem lugar em elas permite a persistência de normas que podem ser mal interpretadas por outros adolescentes ou os mesmos professores da área. Realiza-se uma análise dos principais códigos culturais que se abrangem dentro da linguagem e os vocábulos que utilizam estes jovens como são as normas de convivência grupais, a percepção estética da vida cotidiana, sinais de identidade comuns, representações sociais e prejuízos, estilos de participação social, além do mais dos gestos, as representações sociais e esquematizadas. Também se têm identificado os fatores socioculturais que promovem ou estimulam o abandono escolar dentro da análise general e se têm agrupado em internos (pessoais) e externos (grupais e institucionais).

PALAVRAS CHAVE: sistema simbólico comunitário, capital simbólico, fatores socioculturais, permanência escolar.

PARA CITAR ESTE ARTÍCULO/TO CITE THIS ARTICLE/PARA CITAR ESTE ARTIGO: Leyva Noa, J.J. (2014). La permanencia escolar en las redes educacionales desde comunidades periféricas. Panorama, 8(15), 48-57. 


\section{INTRODUCCIÓN}

El presente trabajo aborda el papel desempeñado por los diferentes actores sociales enmarcados en contextos comunitarios aunque diferentes por razones de diversidad geográfica y cultural, pero sí comunes por el tipo de características congruentes a las comunidades periféricas o también llamadas suburbanas, que por lo general están presentes.

Esta problemática ha sido abordada principalmente desde la pedagogía y la psicología, siendo menos abordada desde los enfoques sociológicos, y muy en especial desde la sociología cultural (Basail y Álvarez, 2004), lo cual se constata a partir de la revisión bibliográfica realizada con los propósitos de la investigación que precedió la redacción de este artículo.

El estudio parte de un acercamiento empírico a la problemática de la permanencia escolar como consecuencia de actos prolongados y definitivos de abandono escolar observado en los estudiantes adolescentes de las comunidades de estudio entre las edades de 12 a 17 años.

Al referirse a la permanencia escolar, muchos autores lo hacen desde la perspectiva de "retención escolar" sin pasar por alto la connotación que el propio término retención adquiere como consecuencia de un acto obligatorio, condicionado o impuesto (Aponte, 1998); otros autores lo valoran como voluntariado e incluso han realizado estudios e investigaciones donde se utiliza este término.

Otros autores agregan que existe una gran variedad de comportamientos denominados con el rótulo común de deserción; pero no deben confundirse con este término todos los actos de abandono en los estudios ni todos los abandonos merecen intervención institucional (Tinto, 1989).

Por lo general, los autores de corte psicopedagógico, en relación con la permanencia escolar, la han tratado desde enfoques cognitivos, y les han atribuido a ellos, en gran medida, el éxito dentro de los currículos, y hecho una equivalencia, aunque no lejos de la realidad que encierran ambos conceptos en cuanto a los objetivos que persiguen. Sin embargo, se ha distinguido poco en cuanto a que la retención escolar encierra una estrategia psicoeducativa que pone en práctica la institución educacional, mientras que en la permanencia escolar, independientemente de que también es tratada dentro de los currículos universitarios, existe en ella una disposición de estar presente una voluntad (Arguedas y Jiménez, 2013).

En México, ya en 1979, aparece un estudio muy extenso donde el fenómeno es identificado bajo el término de abandono. $\mathrm{Al}$ respecto se plantea que ninguna estrategia de intervención aislada será suficiente y que cada universidad debe seleccionar su curso de acción y adoptar diversas medidas (De los Santos, 2004).

Del análisis de la información sobre la permanencia escolar en América Latina, se señala que, en realidad, se trata de un fenómeno que responde a una multiplicidad de factores que se refuerzan mutuamente, evidenciándose en que las condiciones materiales de vida y las características socioculturales de las familias de origen popular determinan el desarrollo de aptitudes y expectativas que no favorecen el éxito escolar y que estas particularidades son reforzadas por un tipo de organización escolar y de prácticas pedagógicas que consolidan las bajas probabilidades derivadas del origen social, conformando un circuito causal que se realimenta constantemente (Tedesco, 1987, p. 6).

La novedad científica del estudio parte de que el tema investigado se ha abordado desde los enfoques socioculturales al tener en cuenta criterios de la sociología cultural.

Los aportes fundamentales están centrados en exponer un modelo teórico conceptual que permita el análisis de la permanencia escolar como un proceso sociocultural a partir de la operacionalización del sistema simbólico comunitario que dio respuesta al objetivo de estudio desarrollado, el cual es aplicable en otros escenarios donde aparezcan problemáticas con características similares (Blumer y Alonso, 1982).

También se determinan los factores socioculturales que condicionan la problemática en estudio a partir de una exploración teórica que se realizó desde las premisas del constructivismo estructural de Pierre Bourdieu, lo que permite la observación de la permanencia escolar como un proceso sociocultural.

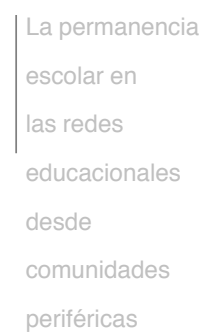




\section{MARCO TEÓRICO}

\section{PRINCIPALES TEORÍAS}

Tras un análisis atento, se halla que las teorías referentes al constructivismo estructural, principalmente lo concerniente al capital simbólico, denominado sistema simbólico comunitario dentro de la investigación al tener en cuenta los referentes culturales planteados por Pierre Bourdieu, y sus nociones de habitus y campo, unido a los requisitos funcionales de toda acción aportados por la teoría funcionalista estructural de Talcott Parsons, permiten relacionar el capital simbólico, los habitus y los campos: por un lado, el campo va a condicionar el habitus; por otro, el habitus le imprime al campo sentido y valor donde debe invertir energía en transformación y adaptación de patrones y normas (Sánchez, 2007). Los babitus constriñen el pensamiento y la acción de los individuos, no los determinan; esta ausencia de determinismo de los habitus permite que el marco teórico aportado en la investigación para el análisis del capital simbólico como requisito funcional para la permanencia escolar no se muestre como un proceso estático, en relación con la diversidad de las características de los estudiantes. Se parte de la concepción parsoniana de los requisitos funcionales de todo sistema de acción: adaptación, consecución de metas, integración y mantenimiento de patrones, para inferir que el capital simbólico constituye un requisito funcional para la permanencia escolar de los estudiantes como proceso sociocultural de adaptación e integración social en la investigación.

Estas teorías suelen optar por un enfoque macroscópico para el estudio de los fenómenos sociales. Al estudiar los grandes sistemas y su interrelación, plantean que las normas institucionalizadas y los procesos culturales son mediados por agentes de control social que permiten la constante socialización de los nuevos individuos (Turner y Turner, 1991), lo cual posibilita, al mismo tiempo, la transmisión del capital simbólico; de esta forma, no obviaban completamente las microestructuras ni la relación entre los actores y las estructuras sociales a niveles macro (Rubio, 2011).

Los interaccionistas simbólicos plantean que las personas aprehenden símbolos y significados en el transcurso de la interacción social al centrarse en una forma específica de interacción social: la socialización (Ritzer, 1992).
En el enfoque conductual, se pone énfasis en las formas de acción e interacción de los individuos dentro de la sociedad y las representaciones sociales, que hacen suyos los diferentes símbolos como parte de un capital simbólico mediante la acumulación de conocimientos que representan realidades sociales.

Por otra parte, la psicología cognitiva, respecto de la adquisición del conocimiento, se ha esforzado en resaltar el papel fundamental de las construcciones mentales y las interpretaciones que los individuos hacen suyas de los significados de los códigos culturales, fundamentalmente en los procesos de comunicación.

En sociología de la educación, se exponen los procesos educacionales como factores de cambio en las estructuras de la sociedad, al concebir la educación como proceso de transmisión cultural y perpetuar el carácter subjetivo de la cultura según el contexto histórico-social, donde tengan lugar las relaciones de intercambio y producción (Quintana, 1993).

La cultura participativa expone que los símbolos son signos que proporcionan un significado por sí mismos; el saber participar se consolida, entre otras cosas, a partir del aprendizaje y la internalización de los símbolos dentro de las redes sociales.

Bourdieu establece una relación directa entre el capital cultural y el simbólico, ya que es la cultura en sí misma la portadora de todos los códigos y las representaciones sociales, que, por medio del aprendizaje y el conocimiento, son descodificados y retransmitidos en los diferentes contextos socioculturales por los grupos sociales; este autor plantea que el capital simbólico, desde el punto de vista cultural, puede existir bajo tres formas: estado incorporado, objetivado e institucionalizado.

A partir de los referentes culturales aportados por Bourdieu, se puede definir el capital simbólico como "un conjunto de códigos culturales aprehendido de los diferentes universos simbólicos como resultado de los procesos de socialización e internalización despegados en cada uno de los contextos socioculturales e individuos transmitido de manera prescripta” (Rubio, 2011, p. 14).

Sobre el sistema simbólico comunitario, la cultura popular participativa lo define como 


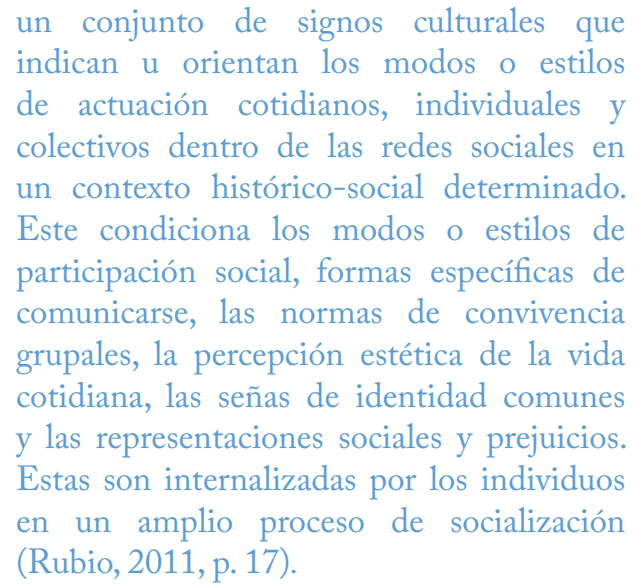

Según el propósito y los enfoques en que está encaminada la investigación, se define el capital simbólico como "el conocimiento acumulado del Sistema Simbólico Comunitario mediante procesos de socialización endógenos"(Rubio, 2011, p. 25).

\section{CONDICIONANTES CULTURALES DE LOS PROCESOS EDUCACIONALES}

La cultura puede ser entendida de muy diversas formas: como civilización, como conjunto de técnicas o procedimientos que requieren conocimiento y habilidad, como conjunto de instituciones y obras "culturales" pertenecientes a un ministerio determinado (museos, música, arte, etc.) o como nivel de instrucción (equivalente a nivel cultural). También se asume la tesis de "la Cultura como sostén de cohesión comunitaria, porque es inobjetable su rol definitivo en los procesos de transformaciones sociales, de las instituciones, organizaciones y grupos" (Espronceda, 2001, p. 38).

El carácter subjetivo de la cultura le permite transmitir sus elementos dentro de la sociedad con facilidad y rapidez desde las estructuras a los individuos por medio del aprendizaje y la socialización, y así, por medio de la cultura, también se controlan las acciones individuales. Es un proceso de construcción y reconstrucción permanente en el que intervienen diferentes factores; sus elementos se encuentran interrelacionados en forma orgánica y poseen la potencialidad de cohesionar y a la vez delimitar o distinguir a diferentes colectivos humanos.

En la actualidad, el pensamiento social responde a favor de los criterios acerca del desarrollo de las culturas locales-regionales, los cuales entran en contradicción con los criterios de diversidad cultural asumidos por las culturas que prevalecen. Por ello, se hace necesaria la comprensión de los procesos culturales comunitarios desde una mirada microsocial (Echevarría, 2002), aun cuando sus alcances funcionales se manifiesten en los niveles de las macroestructuras, como expresara Espronceda (2003, p. 3): "la cultura constituye la estructura que sostiene la cohesión comunitaria, por su rol definitivo en los procesos de transformaciones sociales, de instituciones, organizaciones y grupos". Es así que la cultura que representa el aspecto dinámico de la estructura social, y constituye el alma de las instituciones sociales, viene a ser al propio tiempo el objeto mismo de la educación.

Desde una mirada macrolocal, la influencia del contexto sociocultural en la conducta de las personas permite la fluidez de la cultura a través de los procesos educacionales al desplazar, en determinados momentos, a la educación de su carácter institucional e incorporarla como un proceso social inevitable y constante que va a orientar los modos o estilos de actuación cotidianos, individuales y colectivos dentro de la comunidad. De esta manera, la educación va a llegar a ser masificación de la cultura como expresión de toda forma de enseñanza y aprehensión de conocimientos transmitidos de un individuo a otro fomentando el capital del conocimiento (Tinto, 1989).

Esta influencia de la esfera sociocultural parte desde dos dimensiones en cuanto a espacios geográficos: las zonas rurales y las urbanas, las zonas suburbanas y residenciales, debido a que la población contiene en sí misma toda la diversidad que le concede su estructura: diferencias de género y generación, niveles de instrucción, ocupaciones profesionales o no profesionales, diferencias religiosas, éticas y estéticas.

Desde el punto de vista del espacio geográfico y la dimensión territorial, existe una notada diferencia entre las esferas socioculturales urbanas y suburbanas, independientemente de que los grupos sociales sean los factores claves de todo proceso participativo dentro de cada contexto en relación con el medio social al cual pertenece.

En las comunidades suburbanas con estructuras culturales tradicionales, se observa mayor implicación de los sujetos en procesos de acción comunitaria. Sus puntos de socialización son más definidos que en el contexto urbano, donde los individuos desarrollan su actividad
La permanencia escolar en las redes educacionales desde comunidades periféricas

a


cotidiana en diferentes espacios de socialización; en el caso rural, no se muestra de esa forma, ya que cualquier proceso de cambio afecta, en esencia, el equilibrio de la estructura cultural. Cuando dichas estructuras son permeadas o invadidas por normas culturales institucionalizadas homogeneizantes externas, provoca la marginación social de aquellos sujetos que no poseen una orientación adaptativa al cambio.

Los factores socioculturales van a condicionar, en gran medida, los procesos educacionales, ya que el individuo es operante en la medida en que se dispone a reaccionar con el ambiente que lo rodea. Estos factores van a influir en las conductas asumidas por los individuos que se mueven dentro de diferentes contextos sociales.

Al hablar de factores socioculturales, nos referimos a los agentes sociales que influyen, determinan y condicionan procesos socioculturales comunitarios en diferentes contextos ambientales; ellos facilitan no solo conocer los rasgos distintivos que hacen diferentes a grupos humanos y asentamientos poblacionales dentro de un mismo espacio geográfico y social, sino, además, comprender, a través de ellos, cómo se ha producido el desarrollo histórico, sus tendencias más significativas, lo que posibilita explicar los nexos que se establecen entre las actuaciones de individuos y grupos y las dinámicas sociales (De los Santos, 2004, p. 34). Estos no son dimensiones ni elementos, son condiciones determinantes en cuanto reportan esencialidades de los comportamientos humanos. Aspectos como la religiosidad, las costumbres y las tradiciones aportan un conjunto de significados que no se pueden soslayar en los estudios de las comunidades.

\section{LA PERMANENCLA ESCOLAR DESDE LA PERSPECTIVA SOCIOCULTURAL}

Un rasgo que ha caracterizado a los estudios socioculturales es el de construir teorías generales que permitan articular críticamente diversos escenarios de la vida social al incorporar el enfoque global para explicar los procesos culturales como una perspectiva totalizadora, por lo que los estudios socioculturales rebasan el campo de una disciplina para abordar, desde lo transdisciplinar, los fenómenos culturales (Ander, 1965). Por ello, más que buscar la formación de un campo disciplinario, conforman nuevas formas interpretativas y nuevas formas de significación.
El campo más específico de los estudios socioculturales es el de los estudios sobre la vida cotidiana; y la disciplina más próxima a ella es la etnografía. Dichos estudios consideran aspectos como la diversidad, la cual expresa la distinción y aceptación de aquellos rasgos que signan los comportamientos y maneras de interpretar, presentes en individuos, grupos, minorías, y la diferencia, entendida como proceso de enunciación de una cultura, con lo cual esta deviene conocible y adecuada a la creación de medios de identidad cultural.

Para los estudios socioculturales, la permanencia escolar como concepto adquiere nuevas dimensiones; cada vez más es el proceso donde con mayor nitidez se puede advertir una diversidad de matices, sentimientos, opiniones $\mathrm{y}$, de manera singular, las especifidades de las relaciones sociales dentro de contextos que permitan el flujo cultural como expresión de la educación; las interpretaciones que dan los individuos y grupos a los hechos de asumir o no diferentes posturas ante el estudio de forma voluntaria e involuntaria, así como las propuestas de alternativas para mantener, modificar y transformar la idea de continuar el estudio (Blanco, 1997). Ello significa también que, a través del estudio y análisis de la cultura, se conocen y se comprenden las distintas actitudes y comportamientos humanos y su repercusión en las valoraciones sobre la realidad que presentan.

A través de los estudios socioculturales es posible analizar la permanencia escolar con un enfoque culturológico, el cual pretende visionar el trabajo preventivo-educativo que se desarrolla junto a los factores involucrados en los procesos educacionales como estrategia educativa, y se toman como ejes para el análisis los procesos culturales al caracterizar el nivel de las relaciones que se establecen entre individuos, grupos, sectores y clases sociales alrededor de los diferentes contextos comunitarios y de la sociedad en general.

PERMANENCLA ESCOLAR Y PROCESO SOCIOCULTURAL comunitario en Raposo, Primero de Mayo y SUR ISLETA

El estudio parte de un diagnóstico y análisis realizado en tres comunidades periféricas: Raposo, Primero de Mayo y Sur Isleta, ubicadas en los extremos oeste y sur de la ciudad de Guantánamo, municipio cabecera de la provincia que lleva el mismo nombre en la región oriental de Cuba, donde se constató dentro de las principales 
problemáticas socioculturales actos de retiro y abandono escolar, fundamentalmente en los estudiantes adolescentes entre 12 y 17 años, de los que $10.2 \%$ abandonó los estudios, principalmente, en el tránsito de la enseñanza secundaria a la media superior, lo que coincide también con los traslados desde escuelas primarias ubicadas dentro de un contexto sociocultural suburbano hacia uno centro urbano: la secundaria básica.

Esta problemática es también observada durante el periodo de la secundaria básica al ocurrir dificultades en la permanencia en los centros educacionales de, al menos, un $3.07 \%$ de estudiantes, fundamentalmente los que se trasladaron desde las escuelas primarias, ubicadas dentro del espacio geográfico de las comunidades en estudio, hacia las secundarias básicas ya en el interior, pero no centro de la ciudad, lo que da muestra de posibles inadaptaciones sociales a nuevos escenarios socioculturales.

$\mathrm{Al}$ tener en cuenta la anterior situación problemática, se define el siguiente problema científico: ¿cómo incide el sistema simbólico comunitario en la permanencia escolar de estudiantes adolescentes de 12 a 17 años en las redes educacionales desde comunidades periféricas?

El objetivo general es aportar un modelo conceptual para el análisis de la permanencia escolar en relación con el sistema simbólico comunitario, a fin de comprender la incidencia de los procesos culturales comunitarios en los procesos educacionales en las comunidades Raposo, Primero de Mayo y Sur Isleta.

A partir del problema anterior se propone la siguiente idea por defender: en los procesos educacionales no solo ocurre el aprendizaje de conocimientos académicos, sino también constituyen un escenario propicio para los procesos de socialización de normas éticas y estéticas y de todo el sistema simbólico comunitario heredado y aprendido desde la familia hasta los diferentes contextos socioculturales en que se desarrolla el individuo, por lo que la escuela como institución educacional cumple una función de mediadora cultural entre las normas macroestructuradas y los procesos culturales comunitarios o microestructurados. El capital simbólico, denominado sistema simbólico comunitario, al tener en cuenta los referentes culturales aportados por Bourdieu, condiciona la actuación del individuo dentro y fuera de su contexto cotidiano, aun cuando este pueda ser interpretado o recodificado por el actor con determinada independencia y creatividad. El habitus con el que llegan los estudiantes desde el contexto suburbano es parte de su capital simbólico, pero este no es suficiente para integrarse al nuevo contexto de socialización urbana al que acceden, porque dentro de él coexisten otros habitus que, con el tiempo, necesitan aprehender para garantizar su adaptación dentro de ese nuevo contexto. De esta forma, el capital simbólico portado por los estudiantes que se trasladan desde un contexto educacional y social suburbano hacia uno centro-urbano constituye un requisito funcional para la adaptación e integración del estudiante a la nueva escuela como contexto sociocultural.

La permanencia escolar resulta, entonces, un proceso de inserción social donde debe resolverse la contradicción entre los referentes culturales, de origen y de destino, que orientan al estudiante hacia los modos o estilos de actuación cotidianos, individuales y colectivos, propios del centro educacional urbano; dicho proceso implica no solo la aprehensión de nuevas pautas culturales como requisito de adaptación al cambio, sino también su aporte al nuevo sistema simbólico comunitario. De esta manera, el abandono escolar puede ser el resultado de un proceso de adaptación fallido a un nuevo sistema simbólico comunitario, que tiene lugar en la escuela como espacio de socialización urbana.

El estudio se desarrolla desde una perspectiva metodológica cualitativa, que asume un estudio de caso múltiple desde los estudios inclusivos como estrategia fundamental de investigación. También hace alusión, dentro de este paradigma, a un estudio descriptivo; no obstante, se tiende a la triangulación con otros métodos y técnicas de la investigación sociológica, entre ellos, el método etnográfico, los estudios descriptivos, la observación empírica, la observación participante, la encuesta (cuestionario y entrevista), el análisis de documentos y los informantes claves.

La recogida de datos en la investigación se llevó a cabo en los siguientes escenarios: comunidad Raposo, Escuela Primaria Rafaela Caboverde Chacón, comunidad Primero de Mayo, Escuela Secundaria Básica Regino E. Boti y comunidad Sur Isleta. De igual manera, las unidades de observación utilizadas para la investigación la constituyeron los estudiantes adolescentes de 12 a 17 años que abandonaron los estudios en las comunidades 
tratadas en la investigación, los estudiantes en riesgo proclives al abandono escolar de la Secundaria Básica Regino E. Boti y el Preuniversitario Enrique Soto, las familias de los estudiantes que abandonaron y el proceso de enseñanza-aprendizaje.

El instrumento utilizado para la investigación que se aplicó en la recogida de información fue dinámico, diverso y flexible, tal y como ofrece la metodología cualitativa. El estudio fue longitudinal, que abarcó un periodo de seguimiento de 12 meses, dividido en dos etapas consecutivas: la primera de tres meses que incluyó un sondeo en las áreas de estudio, una revisión exhausta de documentos y entrevistas a personas que tratan desde distintas perspectivas el tema; y la segunda de nueve meses, que incluyó observación empírica propia y descubierta, así como observación participante, recogida de muestra en el área y entrevista a informantes claves. Todo esto se desarrolló en consonancia con estudios anteriores relacionados con la problemática investigada.

\section{ANÁLISIS DE LOS RESULTADOS POR CATEGORÍAS}

El lenguaje y los modos de decir encierran una gama de códigos culturales, símbolos, que guardan un significado; lo que permite que se transmita un mensaje y, al mismo tiempo, se reciba una respuesta que no necesariamente resulta idéntica. Los gestos, las representaciones sociales de toda índole, provocan la misma imagen mental tanto en las personas que lo emiten como en las que lo decepcionan. Dentro de los códigos de comunicación más utilizados por los estudiantes encontramos: “¡está escapao!”, para referirse al más inteligente, a los más inteligentes o a los sobresalientes; " $i m a n$, me vas a volver loco o me vas a partir el cerebro!”, para expresar que no está en condiciones de seguir estudiando, discutiendo algún tema, algo que no comprende y trata de evadir; “ipapa!", saludo a colegas, amigos, personas a las que se les tiene aprecio. Esta diversidad de códigos no se muestra en la escuela de origen, ya que los habitus como estructuras internalizadas se comportan paralelos para todos los estudiantes dentro del mismo campo cultural. Los códigos de comunicación provocan un estímulo tanto en el emisor como en el receptor; ellos permiten ser los estimuladores de sus propias acciones, conductas, formas de relacionarse, toma de decisiones, y así permiten la interacción social entre los grupos humanos y los contextos socioculturales comunitarios. La educación como expresión de transmisión del carácter conservador de la cultura solo es posible a través del proceso de comunicación cargada de signos culturales que permiten condicionar procesos educacionales en diferentes espacios de socialización tanto urbanos como suburbanos, ya que los modos de comunicación como herencias culturales presuponen un proceso de cambio sociocultural y, al mismo tiempo, constituyen factores socioculturales que condicionan la problemática en estudio.

\section{NORMAS DE CONVIVENCIA GRUPALES}

Los rasgos normativos y tradicionales de las comunidades suburbanas repercuten en el desempeño de ciertos papeles tanto del hombre como de la mujer, dentro de la familia, que pueden vulnerar la capacidad de participación en el terreno público y privado, lo que va a estancar la autoestima de los estudiantes. La motivación individual en función de la participación social depende básicamente del tipo de personalidad, de las relaciones familiares, las condiciones materiales de vida y el grado de independencia, pero guarda una estrecha relación con la estimulación, el reconocimiento y el apoyo social por las instituciones e identidades de la colectividad.

La poca calidad de las relaciones de comunicación están determinadas, en primer lugar, por la falta de transmisión de valores de padres a hijos: los padres tienen la intención de sentar las bases para el establecimiento de la confianza indispensable en periodos futuros del desarrollo, tales como la adolescencia, mientras que el hijo intenta alejarse de los patrones comunicativos legados por su familia de origen.

En cuanto a la formación de valores como tendencia general en las familias estudiadas, los padres han dado prioridad al desarrollo de la independencia que debe expresarse en el logro de autonomía económica, en la validación para desempeñar cualquier tarea en el hogar, la defensa de un espacio propio, la capacidad de tomar sus propias decisiones y asumir las consecuencias.

Un rasgo característico en las formas de convivencia son los grupos de pares, los que establecen pautas de carácter hegemónico para con sus miembros, condicionan el estatus de los estudiantes en cuanto a lo que está de moda dentro del grupo, lo que es permitido, lo que es de agrado; ellos definen quién puede permanecer dentro 
de su "grupo de afinidad", si cumple las exigencias de la colectividad que lo integra.

\section{PERCEPCIÓN ESTÉTICA DE LA VIDA COTIDIANA}

La percepción estética de la vida cotidiana implica la manera como los grupos humanos aprecian desde el punto de vista artístico, creativo y diverso, la cultura o el resultado de un producto acabado dentro de las comunidades condicionado culturalmente por factores que promueven o dificultan la acción contextual.

Los miembros de las comunidades y los grupos sociales son representantes culturales auténticos, atendiendo a sus motivaciones, habilidades y gustos específicos, y se erigen potenciales portadores de uno o varios de los elementos que enriquecen el conjunto de valores culturales comunitarios. Esta concepción nos indica un sendero de comprensión de la cultura más allá de los límites de su conceptualización, que se debate entre difusión de conocimientos y desarrollo de la sensibilidad estética a partir de códigos estéticos homogéneos o estandarizados; de esta manera, el desarrollo cultural solo se logra potenciar tomando como punto de partida la creatividad colectiva en toda su diversidad y su promoción participativa. Ello implica ver la cultura no como un resultado por obtener o un producto acabado, posible de ser evaluado únicamente mediante indicadores cuantitativos, sino como procesos que se generan desde y por los grupos sociales, a partir de intercambios simbólicos: saberes, diversas formas de comunicación, de prácticas cotidianas, que cada comunidad o grupo social construye, se apropia, reproduce y transforma, en forma dinámica, para conformar su identidad.

Muchos de estos estudiantes están colmados de pesimismo e imaginan un futuro incierto donde tienen que cargar con el modelo representativo de las familias en la sociedad: "qué importa estudiar si de todas maneras tengo que ayudar en el campo", "no me gusta estudiar y no me importa quedarme bruto" o "como si la vida fuera color de rosa"; no buscan una superación cultural, sino una remuneración económica, aun cuando su nivel de instrucción esté por debajo de los que los rodean; ello conlleva necesariamente el fracaso social.

\section{SEÑAS DE IDENTIDAD COMUNES}

En la mayoría de los casos, la formación de la identidad se vincula con el género de modo que se refuerzan los estereotipos masculinos y femeninos. A los hijos se les enseña a ser independientes, decididos, seguros de sí mismos, promiscuos, eficientes en los espacios públicos, mientras que en las hembras se promueve la delicadeza, el desarrollo de competencias en el área doméstica, y se obvian temas como la sexualidad, que resultan tabú en las relaciones de padres e hijos, lo que manifiesta la existencia de diferencias de género que marcan el desempeño de la función socializadora. Coinciden en la educación de ambos géneros el desarrollo del valor de la independencia, lo cual se contrapone en las hembras con el papel tradicional: la reproducción, el cuidado del hogar, la complementación en el sexo. Sobre la formación de la identidad, los hijos replican los papeles que desempeñan los adultos en el hogar y esto se refuerza con una educación estereotipada relativa al género.

Aunque este sistema simbólico sea interpretado por los estudiantes con determinada independencia, y permita cierta contribución a su desarrollo, siempre va a condicionar la actuación de los estudiantes dentro y fuera de su contexto cotidiano. El conocimiento del sistema simbólico permite a los estudiantes identificar las señas de diversidad cultural y los niveles de sensibilidad comunitaria.

Otro elemento por evaluar es lo concerniente a la manera no fluida de hablar, de usar el uniforme, las preferencias recreativas; todo esto distingue fuera de su contexto cotidiano (la comunidad suburbana y la escuela) a los estudiantes observados. Formas de expresarse como "tengo el estómago en la nave de voltus $\mathrm{V}$, perdida en el Olimpo" para referirse a la sensación de hambre, “¡ahí, ahí!", para destacar arrogancia por algo que habían advertido y no lo habían tenido en cuenta, la no fluidez a la hora de hablar dificulta la comunicación, muchas veces no entienden lo que se les dice y hay que repetirles la idea, se deben fundamentalmente a una mala expresión oral y escrita desde la familia, donde, según las entrevistas, sus padres no supieron asumir los papeles convenientes en la primaria formación de sus hijos.

\section{REPRESENTACIONES SOCLALES Y PREJUICIOS}

De los estereotipos sociales, el más problemático se relaciona con la división del trabajo por género dentro del hogar. La actividad representativa de la mujer consiste en el trabajo reproductivo, productivo y comunal,

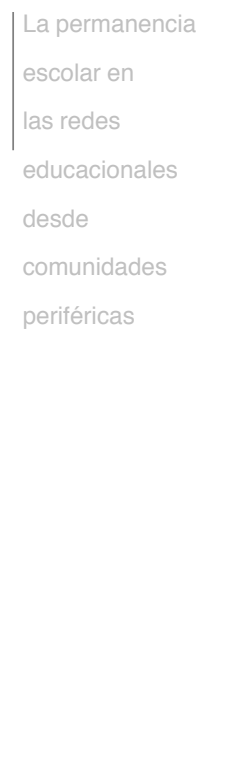


mientras que en el hombre varía el contenido de tales labores, que van desde la responsabilidad del hogar, el trabajo forzado y la toma de decisiones. De ahí que los debates predominantemente feministas se tracen la problemática central del triple papel, en el que nace el poder y la opresión en relaciones de género y términos de la subordinación de la mujer al hombre, de ahí que se atribuye las mayores cifras de variabilidad de la permanencia escolar al sexo femenino, sosteniendo el papel de la mujer en cuanto a la reproducción a temprana edad y la aceptación de papeles ya enraizados culturalmente dentro de los hogares que responden a ciertos modos de participación social.

No es sorprendente encontrar criterios entre los estudiantes que provienen de comunidades suburbanas hacia urbanas, como “ila novela, eso es de mujeres!” " “ila mujer es para estar dentro de la casa”, "ino le des mucho hilo que se te monta!", entre otros más donde prima el autoritarismo masculino.

\section{MODOS O ESTILOS DE PARTICIPACIÓN SOCIAL}

Los modos o estilos de participación social son el resultado de un aprendizaje en la colectividad como en la individualidad dentro del contexto sociocultural comunitario. Las acciones impuestas a los estudiantes en estudio desde el exterior del medio comunitario provocan un cambio mecánico e impersonal, y la tendencia social es el rechazo; sin embargo, las acciones propuestas en el interior del medio comunitario son aceptadas, enriquecidas y sostenidas por los individuos. La participación social en los estudiantes es el resultado del desarrollo de mecanismos dinámicos, adaptativos y creativos, internalizados por estos para poder existir y manifestarse, lo que implicó indiscutiblemente un amplio proceso de interacción y socialización, mediante el cual han aprendido las maneras de actuar en un determinado grupo o comunidad al adquirir códigos, símbolos y herramientas morales fundamentales para interactuar en uno $\mathrm{u}$ otro campo cultural.

Todo grupo social posee una autonomía simbólica, muestra un conjunto de signos que lo autodefinen en un sentido cultural, lo cual se expresa en los modos o estilos de vida que condicionan un tipo específico de comunicación grupal y de espacios de sociabilidad que, en consecuencia, le permiten actuar en contextos socia-
La participación social presupone un aprendizaje de códigos y normas de conducta, el cual conforma y regula los rasgos personológicos distintivos dentro de un entorno sociocultural delimitado. Las representaciones sociales de los estudiantes a través de los estilos o las formas de representación social permiten que ellos no se automarginen, que se sienta inferiores, incapaces, ajenos a lo que sucede a su alrededor.

\section{CONCLUSIONES}

La permanencia escolar no está causada por deficiencias en el proceso de formación de los estudiantes en las escuelas, aun cuando lo concerniente a los métodos y teorías de enseñanza que circulan en las instituciones privilegia los aprendizajes cognitivos por sobre los socioafectivos, sino que está mediada por factores socioculturales.

La concepción del problema está puramente desde lo externo de los procesos educacionales, donde influyen otros factores que no son propios del sistema educacional, sino de las herramientas culturales que han aprehendido estos estudiantes de los diferentes contextos socioculturales en los que se han desarrollado.

Independientemente de que el proceso educativo analizado se muestre eficaz, su debilidad radica en que no tiene en cuenta el capital simbólico portado por los estudiantes que ingresan de sectores sociales suburbanos como punto de partida de los procesos de enseñanza-aprendizaje.

Los habitus con los que llegan los estudiantes adolescentes desde los contextos suburbanos son parte de su capital simbólico, los que pueden ser suficientes e insuficientes, necesarios e innecesarios. Ellos deben ser adaptados a los nuevos contextos de socialización urbana a los que acceden, porque en ellos coexisten otros habitus, los que van a condicionar una inserción a las normas prevalecientes como requisito de adaptación e integración social.

Los factores socioculturales van a influir en las actitudes de los estudiantes adolescentes, debido a que los individuos son operantes en la medida en que se disponen a reaccionar según las peculiaridades del contexto sociocultural en el que se encuentren; estos 
influyen en la permanencia escolar de estudiantes adolescentes, los que se clasificaron para una mejor comprensión en internos (personales) y externos (familiares e institucionales).

\section{REFERENCIAS BIBLIOGRÁFICAS}

1. Aponte, E. (1988). Perfil del desertor escolar y estrategias para retenerlo. Pedagog $\varphi$ a, 25(2), $55-65$.

2. Ander Egg, E. (1965). Técnicas de investigación social. México: Editorial Humanitas.

3. Arguedas Negrini, I. y Jiménez Segura, F. (2013). Factores que promueven la permanencia de estudiantes en la educación secundaria. Revista Actualidades Investigativas en Educación, 7(3), 1-36.

4. Blanco, A. (1997). Introducción a la sociología de la educación. La Habana: Facultad de Ciencias de la Educación/Instituto Superior Pedagógico Enrique José Varona.

5. Basail, A. y Álvarez, D. (2004). Sociología de la cultura. La Habana: Editorial Félix Varela.

6. Bourdieu, P. (1990). Sociología y cultura. México: Grijalbo.

7. Blumer, H. y Alonso, P. R.(1982). El interaccionismo simbólico: perspectiva y método. Barcelona: Hora.

8. Cabanas, J. M. Q. (2004). La educación está enferma: informe pedagógico sobre la educación actual. Nau llibres.

9. De los Santos, E. (2004). Los procesos de permanencia y abandono escolar en educación superior. Revista Iberoamericana de Educación, 3(12), 1-7.

10. Espronceda Amor, M. E. (2001). Parentesco, inmigración y comunidad. Una visión del caso haitiano. Guantánamo: El Mary La Montaña.

11. Espíndola, E.y León, A. (2002). La deserción escolar en América Latina: un tema prioritario para la agenda regional. Revista Iberoamericana de Educación, 30, 39-62.

12. Echeverría, L. D. (2002). La dimensión cultural del desarrollo para el trabajo comunitario. Matanzas, Cuba: Consejo Científico de Cultura en Matanzas.

13. García, J. y Lázaro, J. (1997). Sociología de la cultura. México: Paidós.
14. Gonzáles, D. (1992). Psicología educativa. La Habana: Editorial Pueblo y Educación.

15. Jíbara, L. (1998). Psicología y educación. La Habana: Editorial Pueblo y Educación.

16. Narodowski, M. (1996). Enfrentar la deserción escolar. Universidad Autónoma de Entre Ríos.

17. Piaget,J. (1972). Psicología y pedagogía. Barcelona: Ariel.

18. Quintana Cabanas, J. M. (1993). Pedagogía familiar.

19. Ritzer, G. (1992). Teoría sociológica clásica (2. ed.). Barcelona: Editorial Buena Vista.

20. Rosales, A. (2007). Retención escolar en los ISP y deserción escolar en las carreras de lenguas extranjeras. Guantánamo: ISP Raúl Gómez García.

21. Rubio Méndez, D. (2008). Cultura participativa y sistema simbólico comunitario. Santiago de Cuba: Universidad de Oriente/Facultad de Ciencias Sociales.

22. Rubio Méndez, D. (2011). Análisis crítico de la teoría de los estados del capital cultural de Bourdieu. Santiago de Cuba: Universidad de Oriente/ Facultad de Ciencias Sociales.

23. Sánchez, R. (2007). La teoría de los campos de Bourdieu como esquema teórico de análisis del proceso de graduación en posgrado. Revista Electrónica de Investigación Educativa, 9(1).

24. Tinto, V. (1989). La deserción en la educación superior: síntesis de las bases teóricas de las investigaciones recientes. Review of Educational Research, 45(1), 89-195.

25. Tedesco, J. C. (1987). El desafío educativo. Buenos Aires: Grupo Editor Latinoamericano.

26. Turner,J. H. y Turner, P. R. (1991). The structure of sociological theory (5. ${ }^{\mathrm{a}}$ ed.). Belmont, CA: Wadsworth. 